\title{
Estimating crustal parameters using joint inversion of receiver-function and gravity data
}

\author{
Yawei Ma ${ }^{1}$, Lianghui Guo ${ }^{2}$ \\ School of Geophysics and Information Technology \\ China University of Geosciences \\ Beijing, China \\ myw@cugb.edu.cn
}

\author{
Lei $\mathrm{Shi}^{3}$ \\ Institute of Geophysics, China Earthquake Administration \\ Beijing, China \\ Shilei_cugb@163.com
}

\begin{abstract}
In the exploitation of the mineral resources, deep subsurface structure research can explain the formation mechanism of resources and guide proper resource assessment. Receiver-function is an effective method to solve problems related to deep structure. Based on the uncertainty of this method, which relies on data quality, a kind of constraint should be introduced. The observed gravity anomalies are mainly composed of crustal gravity and Moho gravity. The former is closely related to crustal density $(\sigma)$ and velocity ratio $(k), v_{p} / v_{s}$, while the latter is related to crustal thickness $(H)$. Therefore, gravity inversion can provide $H, \sigma$ and $k$, furthermore, it can constrain the $H-k$ stacking, to guarantee the computational precision. Testing on the model data, we found that this method has an effective constraint for $\boldsymbol{H}-\boldsymbol{k}$ stacking of seismic receiverfunction amplitudes and it can be used for real data.
\end{abstract}

Keywords-gravity inversion; receiver-function; H-k stacking; maximum likelihood method

\section{INTRODUCTION}

Crustal thickness $(H)$, density $(\sigma)$ and the ratio $v_{p} / v_{s}(k)$ are three important parameters for studying crustal composition and geodynamic process. The $H-k$ stacking technique in receiver-function analysis is popular for estimating $H$ and $k$ parameters ${ }^{[1,2]}$, and has been widely applied in many places. However, in some places, due to the possible shallow-layer unstableness of seismograph stations, complicated tectonics below stations or poor waveform data, multiple reflection phases of receiver-function may be unclear to be identified, which leads to being difficult to pick $H$ and $k$ parameters from the $H-k$ stacking map. The observed gravity anomalies are mainly composed of crustal gravity anomalies caused by uneven density bodies in the crust, and Moho gravity anomalies caused by fluctuation of Moho surface. The former is closely related to $\sigma$ and $k$, while the latter is related to $H$. Hence, gravity inversion can provide $H, \sigma$ and $k$ parameters and thus it can constrain the $H$ - $k$ stacking.

Due to poor quality of receive functions, multiple geophysical methods joint inversion were put forward to estimate the crust parameters. Based on Steiner's (1980) maximum likelihood, Silva and Cutrim assumed that the noise of the observed gravity anomalies obeyed the Gaussian distribution and they put forward a maximum likelihood method of inversion of gravity anomaly ${ }^{[3]}$, which could be used for estimating the geometric parameters of underground geological body. Anthony R Lowry and Marta Pérez-Gussinyé presented a procedure based on integrated analysis of receiverfunction, gravity data and geothermal data for improving estimation of $H$ and $k$ parameters ${ }^{[4]}$.

As a result of unapparent multiple waves, however, we can't obtain the correct $H$ and $k$ from some receiver-functions. Based on Lowry's procedure, we further study the integrated analysis of receiver-function and gravity data for estimating crustal parameters. Differ from their procedure, for those stations are difficult to pick the initial $H$ and $k$ values, we estimate their Moho depths via gravity anomaly separation and interface inversion, and then transform them into the initial $H$ values, and then guide to pick the initial $k$ values from $H-k$ stacking map.

In this paper, we apply two data sets, respectively, as the $H$ and $k$ forward gravity anomalies. Then we select several points in the region as station location to forward receiver-functions. In order to highlight the feasibility and effectiveness of the method, we set the weighting factors of Ps to 1 when stacking receiver-functions. After that, we multiply stacking result by maximum likelihood result together to achieve a more accurate result of the $H$ and $k$.

\section{Methodology}

The purpose of joint inversion is solving the problem, which underground discontinuities that can't be calculated owing to poor quality of receiver-function. The procedure of the joint inversion technique is presented in the following:

Step 1, pick up one station that we can't calculate a correct result by using $H-k$ parameter stacks. And search other stations and gravity anomaly data around this station.

Step 2, collect regional geological and geophysical data as constraint conditions and invert $H$ by using constant density interface depth inversion method ${ }^{[5,6]}$.

Step 3, search for all stations to obtain $H$ and $k$ parameters in this area, then mesh them into the same size with gravity anomaly.

Step 4, using the gridding $H$ and $k$ as initial value, calculate $\Delta \rho_{\text {Moho }}$ and $\partial \rho_{\text {Crust }} / \partial k$ via next expression: 


$$
\begin{gathered}
\Delta g_{\text {Moho }}=B_{H} \cdot \Delta \rho_{\text {Moho }} \\
\Delta g_{\text {Crust }}=B_{k} \cdot \partial \rho_{\text {Crust }} / \partial k
\end{gathered}
$$

where

$$
\begin{gathered}
B_{H}=F^{-1}\left\{2 \pi G \cdot F\{H-\bar{H}\} \cdot e^{-f \bar{H}}\right\} \\
B_{k}=F^{-1}\left\{2 \pi G\left[\frac{1-e^{-f \bar{H}}}{f} F\{k-\bar{k}\}-c \cdot e^{-f \bar{H}}\right]\right\} .
\end{gathered}
$$

Here $\Delta \rho_{\text {Moho }}$ is the Moho density contrast. $B_{H}$ is amplitudes of the Bouguer per unit density, which associated with $H . \bar{H}$ is the mean value of $H . F\{\bullet\}$ and $F^{-1}\{\bullet\}$ denotes the Fourier transform operator and Fourier inversion transform operator respectively. $G$ is the universal gravitational constant and $f$ means wave number in frequency domain. $\partial \rho_{\text {Crust }} / \partial k$ is the partial derivative of density to k. $B_{k}$ is the Bouguer per unit partial derivative amplitudes associated with $k$. And $\bar{k}$ is the mean value of $\mathrm{k} . \mathrm{c}=F\{(H-\bar{H})(k-\bar{k})\}$ is a correction factor for variable crustal thickness[4].

The $\Delta \rho_{\text {Moho }}$ and $\partial \rho_{\text {Crust }} / \partial k$ can be obtained via next expression:

$$
\Delta g_{B}=\Delta g_{\text {Moho }}+\Delta g_{\text {Crust }}
$$

Step 5 , calculate modelled gravity filed by using $\Delta \rho_{\text {Moho }}$ and $\partial \rho_{\text {Crust }} / \partial k$ in (5).

Step 6, subtract the modelled gravity from observed gravity as the residual gravity filed. According to Silva and Cutrim, assuming the residual gravity obey the Gaussian distribution ${ }^{[3]}$, we can obtain the best mean and variance by adopting maximum likelihood method.

Step 7, change $H$ and $k$ located center station in a same parameter space with receiver-function stacking. Calculate residual gravity anomaly and a likelihood value of the $(H, k)$ parameter space at the center site using expression next:

$$
y=f(x \mid \mu, \sigma)=\frac{1}{\sigma \sqrt{2 \pi}} e^{\frac{-(x-\mu)^{2}}{2 \sigma^{2}}} .
$$

Step 8, multiply stacking result by maximum likelihood result together, and pick up the best estimate of $(H, k)$.

Step 9, return to step 3 and replace $(H, k)$ with new $(H, k)$. Recalculate from step 3 to step 8 till the precision $(\Delta H)$ is less than $0.1 \mathrm{~km}$. The $\Delta H$ is easily shown that

$$
\Delta H=H_{n}-H_{n+1} .
$$

\section{MODEL DATA EXPERIMENT}

Theoretical model requires crustal thickness close to reality, so the crustal thickness need to be smoother, meanwhile, $v_{p} / v_{s}$ should reflect some local information. Now, assuming altitude is $0 \mathrm{~m}$, so the depth of Moho and the thickness of the crust are the same. The model of $H$ and $k$ are showed in Fig.1 and Fig. 2 respectively. The mean of $H$ is $40 \mathrm{~km}$ and the mean of $k$ is 1.7 .

We choose $\Delta \rho_{\text {Moho }}=0.23 \mathrm{~g} / \mathrm{cm}^{3}$ and in $\partial \rho_{\text {Crust }} / \partial k=0.25$ in setting up (1) and (2) respectively [4]. The Bouguer gravity anomaly is computed by Parker's method. The result is shown in Figure 3.

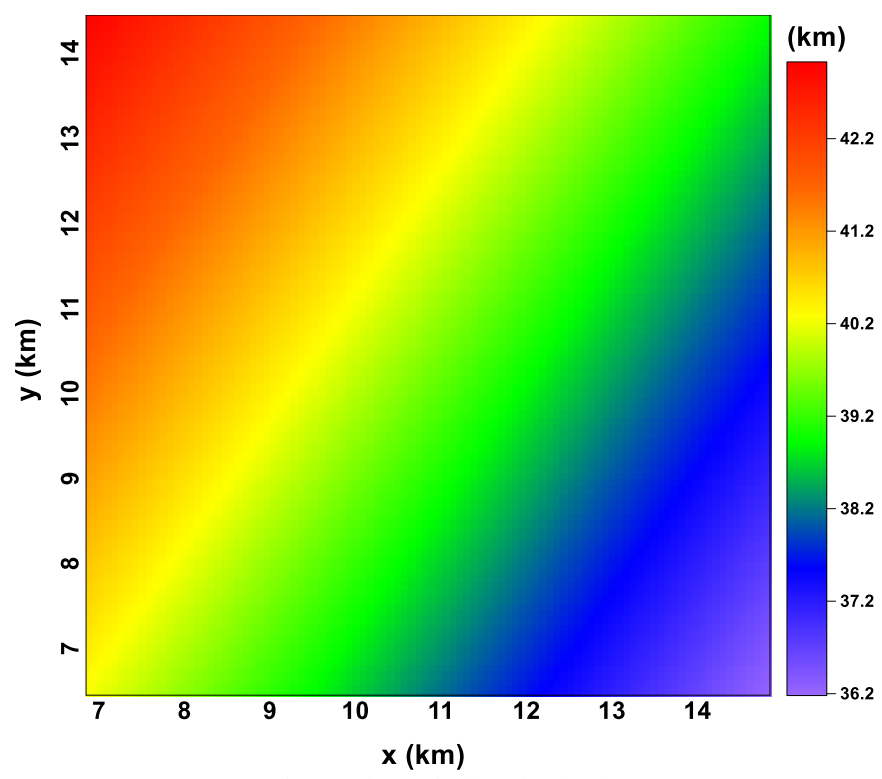

Fig. 1. Theoretical Moho depth

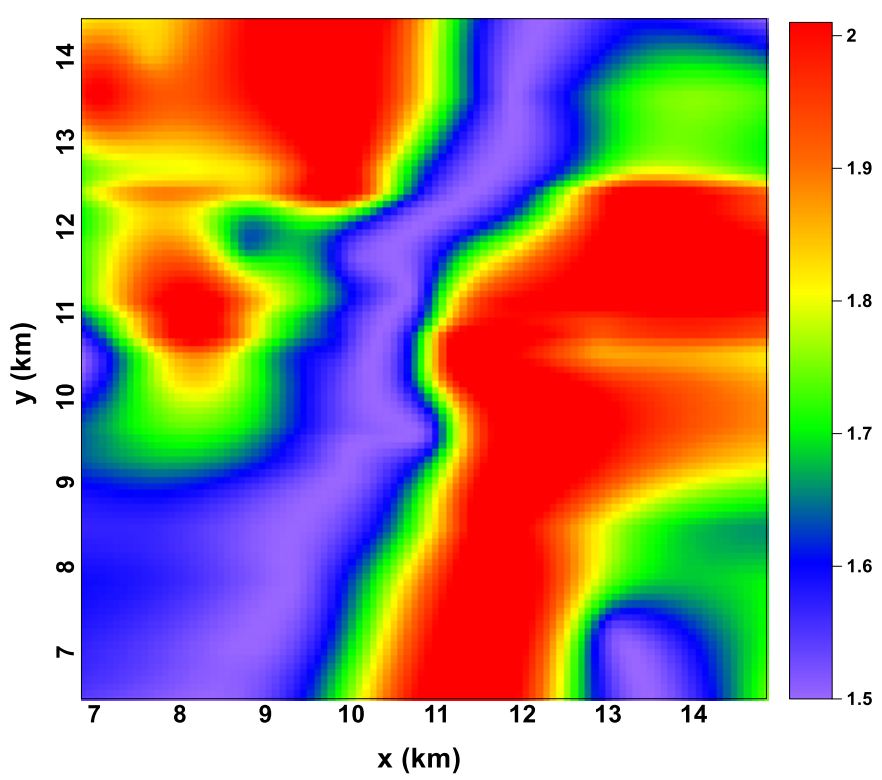

Fig. 2. Theoretical $v_{p} / v_{s}$ ratio 


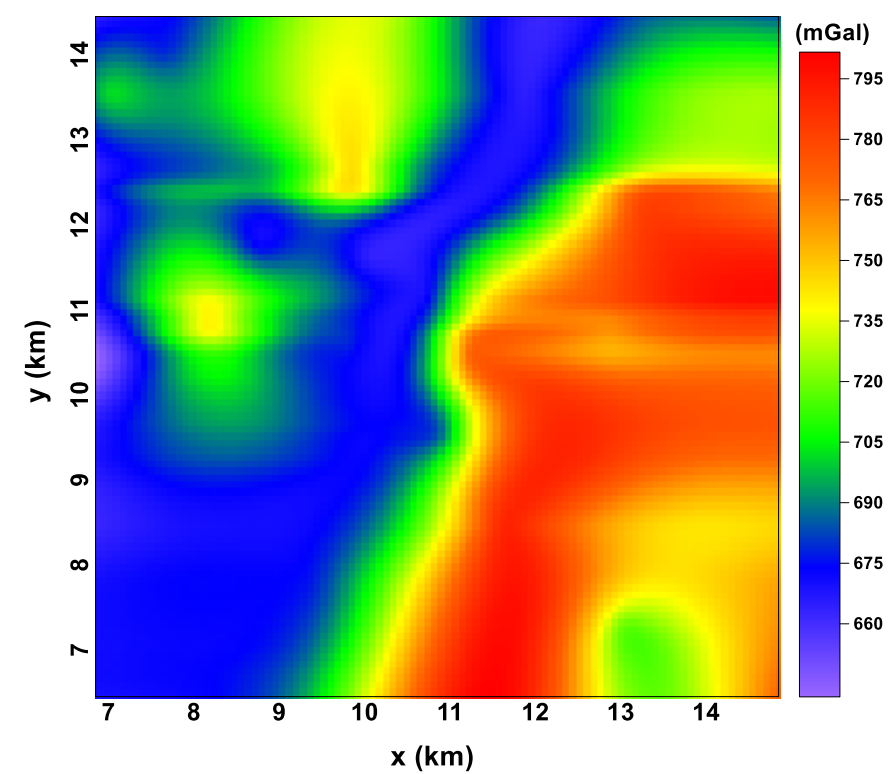

Fig. 3. Theoretical gravity anomalies

It can be found that the anomalies caused by Moho mainly embodied in region, and crustal gravity anomalies that associated to the $v_{p} / v_{s}$ embodied in local. We map the gravity anomalies after adding Gaussian noise (Fig.4).

Here, Fig.4 is the map of initial anomalies, we can estimate $\mathrm{H}$ and $\mathrm{k}$ by maximum likelihood method. The likelihood estimation values shown in Fig.5. There is a maximum likelihood value in Figure 5, but the effective estimation is still in a larger scope.

Now we forward receiver-functions using depth in central Fig.1. Assuming $v_{p}=6.1 \mathrm{~km} / \mathrm{s}$, and the ray parameter ranges from 0.045 to 0.080 . There, every ray parameter meaning different earthquake event. We can get eight receiverfunctions from these events (Fig.6).

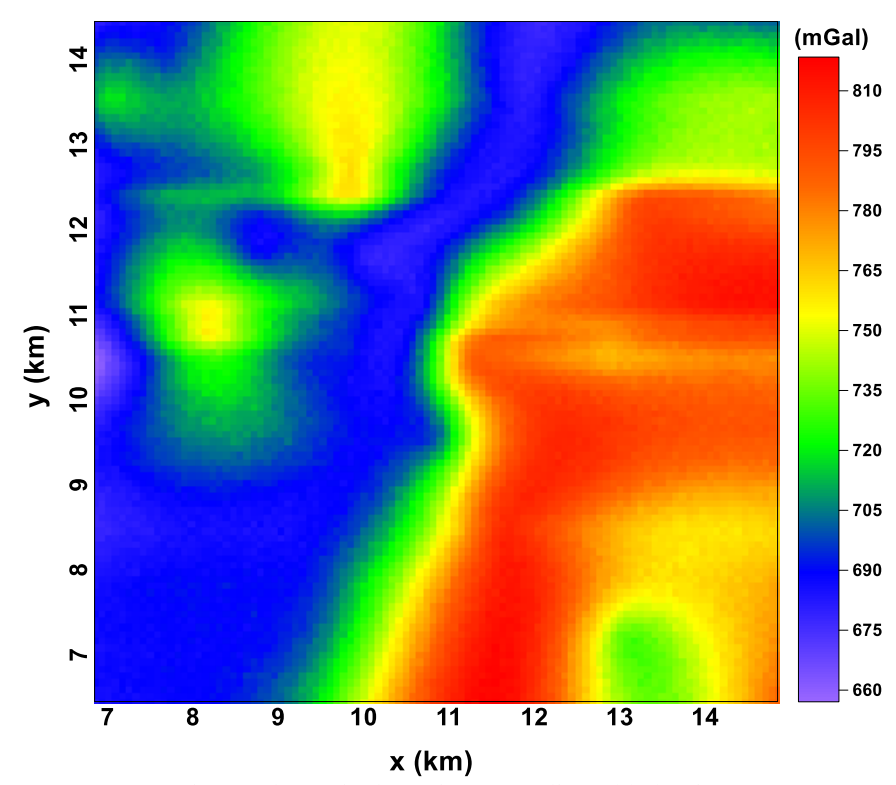

Fig. 4. Theoretical gravity anomalies added noise

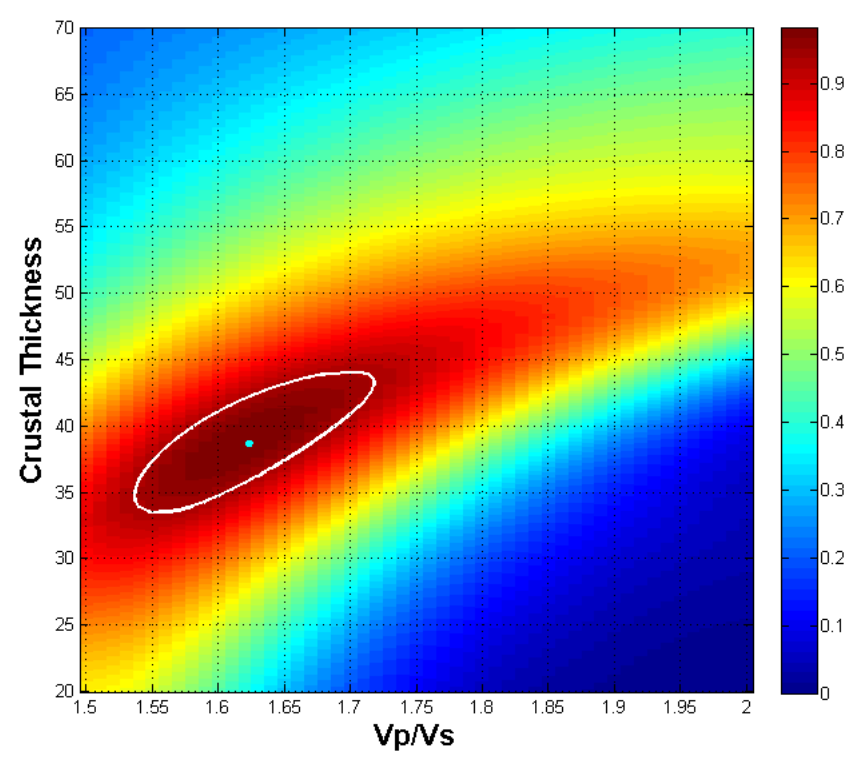

Fig. 5. Gravity maximum likelihood spectrograms

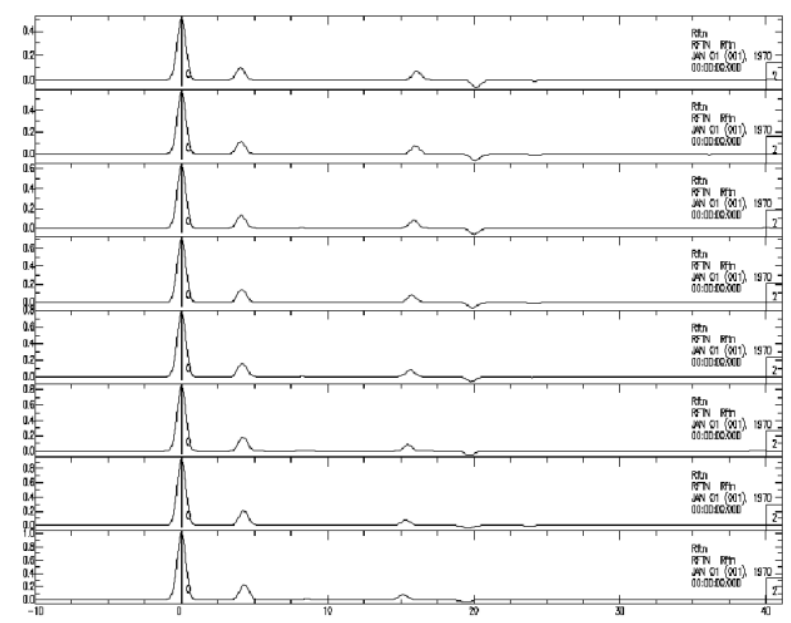

Fig. 6. Theoretical receiver-functions

Obviously, besides the first breaks, there are three multiple waves in each of theory receiver-functions. These waves are Ps, PpPs and PsPs+PpSs. However, it is usually difficult for multiple waves to appear clearly in the actual data. Mostly there are only $\mathrm{Pp}$ and Ps. In order to be close to the real problems, we set the weighting factors of Ps to 1 when stacking receiver-functions. Stacking result is given in Figure 7. We can find that there is a maximum. However, the best estimate value of $H$ and $k$ lies in a range rather than a determined value. This is the problem we have proposed before. In some cases, receiver-function has no idea to derive an answer that we need. We need both gravity and receiverfunction play a role.

Multiply $H-k$ parameter amplitude stacks results by maximum likelihood results together. Now the likelihood filter added gravity constraint shown in Figure 8. 


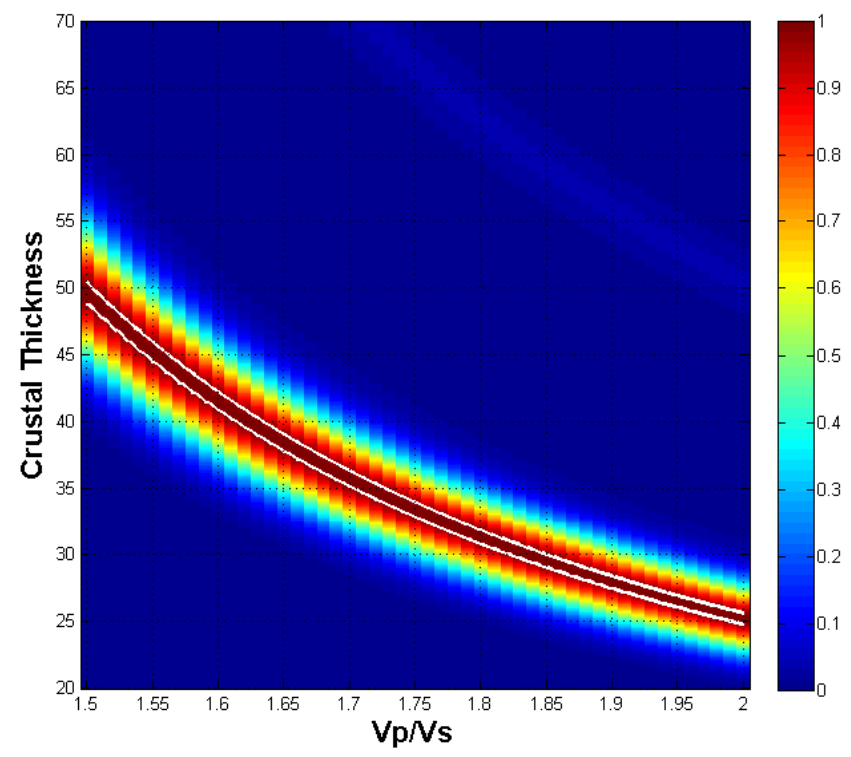

Fig. 7. $H-k$ stacking

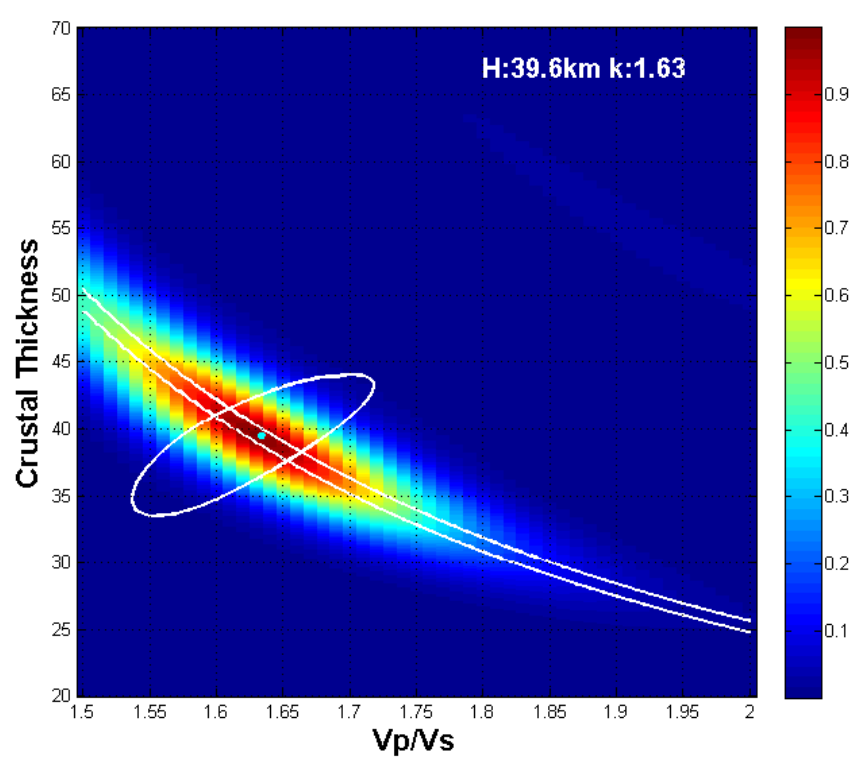

Fig. 8. Likelihood spectrograms of gravity and receiver-functions

As shown in Fig.8, receiver-function still plays a major role. Adding the constraint of gravity leads estimated value to become more accurate. The calculated value of $H$ is $39.6 \mathrm{~km}$ and the value of $k$ is 1.63 in Figure 8 . The $H$ of starting model center site is $39.62 \mathrm{~km}$ and the $\mathrm{k}$ is 1.63 beneath the site. The error of the $H$ between calculated result and model is only 200 meters. In $H-k$ scanning process, we set interval of the $H$ to $0.1 \mathrm{~km}$, so the error $(<0.1 \mathrm{~km})$ is acceptable.

\section{CONCLUSIONS}

Based on the gravity anomaly constraint, we invert crustal thickness and $v_{p} / v_{s}$, and validate the method through different models. The forward results are added noises sequentially from $1 \%$ to $5 \%$ of the maximum. We present the priciple and procedure of the joint technique in details. A series of experiments show that this method can be used for estimating the crustal thickness and the ratio $v_{p} / v_{s}$ within the appropriate range of parameters.

With $H$ from the gravity anomaly separation and interface inversion, this method can picks the initial $k$ from $H-k$ stacking map. It improves the computational precision by the iteration of $(H, k)$ and solves the problems that receiver-function can't solve unilaterally.

\section{ACKNOWLEDGMENT}

This work was financially supported by the National Natural Science Foundation of China (41374093, 41404063), and the Fundamental Research Funds for the Central Universities (2652015214).

\section{REFERENCES}

[1] L.P. Zhu, "Moho depth variation in southern California from teleseismic receiver-functions". Journal of Geophysical Research, vol, 105, pp. 2969-2980. 2000.

[2] X.B. Tian, Zhang, Z.J., "Bulk crustal properties in NE Tibet and their implications for deformation model". Gondwana Research, vol, 24, pp. 548- 559. 2013

[3] J.B.C Silva, A.O. Cutrim, "A robust maximum likelyhood method for gravity and magnetic interpretation". Geoexploration, vol, 26, pp.1-31. July 1989.

[4] A.R. Lowry, M. Pérez-Gussinyé., "The role of crustal quartz in controlling Cordilleran deformation". Nature, 471, pp. 353-357. 2011.

[5] R.L. Parker, "The Rapid Calculation of Potential Anomalies". Geophys, vol, 31, pp. 447-455. 1972.

[6] Zhang Sheng, X.H. Meng, "Constraint interface inversion with variable density model". Progress in Geophys, (in Chinese), vol, 28, pp. 17141720. 2013 